\title{
Small bowel obstruction with multiple perforations due to enterolith (bezoar) formed without gastrointestinal pathology
}

\author{
Aws S. Salim \\ Department of Surgery, The Royal Infirmary, Perth, PHI INX, UK.
}

\begin{abstract}
Summary: An enterolith (bezoar) usually originates in an intestinal diverticulum or in a segment of bowel loculated by stricture formation. Stasis promotes its formation. This communication describes a case in which a large enterolith caused obstruction and multiple perforations of the terminal ileum in the absence of any predisposing gastrointestinal pathology. The management of this rare occurrence is discussed and the literature reviewed.
\end{abstract}

\section{Introduction}

An enterolith (bezoar) usually originates in an intestinal diverticulum or in a segment of bowel loculated by stricture formation. ${ }^{1}$ Stasis, caused partly by the presence of undigested vegetable material and partly by disordered jejunal peristalsis, ${ }^{2,3}$ promotes the accumulation of bile and the multiplication of bacteria. The bacteria convert cholic acid to insoluble deoxycholic acid and also split glycine and taurine from the bile salts resulting in the precipitation of these materials around some nucleus such as a plum stone. ${ }^{2}$

This communication describes a case in which a large enterolith caused obstruction and multiple perforations of the terminal ileum in the absence of any predisposing gastrointestinal pathology.

\section{Case report}

A 69 year old woman was admitted to hospital with a 4-day history of nausea, vomiting and abdominal pain. Initially the pain was colicky and paraumbilical with a more constant right iliac fossa ache, but after 3 days it became severe and generalized. Her previous history was unremarkable.

On examination she was confused, in some distress and slightly flushed with a temperature of $37.7^{\circ} \mathrm{C}$. She was clinically dehydrated. Her pulse was $98 / \mathrm{min}$ with blood pressure of $100 / 70 \mathrm{mmHg}$.

Correspondence: A.S. Salim, Ph.D.(Surg), F.R.C.S.Ed., F.R.C.S.Glasg, F.I.C.S. The Department of Surgery, Ward 6, Stobhill General Hospital, Glasgow, G21 3UW, UK.

Accepted: 25 April 1990
Abdominal examination revealed generalized tenderness with guarding. A full blood count showed a normal haemoglobin $(14 \mathrm{~g} / \mathrm{dl})$ but a raised white cell count at $18.5 \times 10^{9} / 1$ with $90 \%$ neutrophils. Blood, urea and electrolytes showed changes consistent with dehydration. Liver function tests, serum amylase and blood sugar were within normal limits. Her chest $\mathrm{X}$-ray and electrocardiograph demonstrated no abnormality. Abdominal radiographs revealed evidence of a distended small bowel and intraperitoneal gas. After appropriate resuscitation and a single dose of three antibiotics given intravenously at induction of anaesthesia (piperacillin $4 \mathrm{~g}$; gentamicin $80 \mathrm{mg}$; metronidazole $500 \mathrm{mg}$ ), an emergency laparotomy was performed.

At operation there was copious purulent fluid in the peritoneal cavity, a $20 \mathrm{~cm}$ grossly dilated and congested segment of distal ileum with patchy ischaemia and 3 perforations at necrotic points, and fluid-filled, oedematous, distended loops of proximal small bowel. Palpation revealed a hard, $2.5 \mathrm{~cm}$ intraluminal mass, $30 \mathrm{~cm}$ distal to the gangrenous segment and $25 \mathrm{~cm}$ proximal to the ileocaecal junction. Apart from diverticulae of the sigmoid colon, no other abnormality was detected within the peritoneal cavity. The gallbladder, common bile duct, stomach and duodenum were normal and no diverticulae were seen in the small bowel. The enterolith was milked into the infarcted segment of small bowel which was then resected and an end-to-end anastomosis performed. The abdomen was closed after thorough Noxyflex peritoneal toilet. The patient made an uneventful recovery. She was well when seen after 6 weeks in the surgical follow-up clinic.

Laboratory analysis of the stone confirmed that 
it was an enterolith and histology examination of the resected segment of intestine showed acute inflammatory changes with ischaemic perforations of the wall. No evidence of any other pathology was detected.

\section{Discussion}

Causes of intraluminal intestinal obstruction include enteroliths (bezoars), which are usually concretions of vegetable matter and food, parasites and gallstones. ${ }^{4,5}$ Rare causes include ginger or drug enteroliths, ${ }^{6}$ or enteroliths formed in duodenal diverticulae. ${ }^{7}$ Enzymic digestion of gastric bezoars has also given rise to intestinal obstruction. $^{8}$ Although more common after gastric surgery, obstruction of the intestine due to enteroliths can occur in the absence of an operation.

Obstruction of the small intestine by an enterolith from a diverticulum situated in the proximal small intestine is rare. ${ }^{9}$ The condition was first reported by Philips in 1921 and a recent review had collected only 25 cases. ${ }^{7}$ Bewes and co-workers reported the formation of a bile acid enterolith in a jejunal diverticulum ${ }^{2}$ and afterwards this was similarly reported by King and associates. ${ }^{10}$ More recently Deutsch et al. ${ }^{11}$ presented the first report of partial small bowel obstruction caused by a gastric cancer bezoar.

Enteroliths tend to remain in the diverticulae in which they are formed. ${ }^{2,3}$ They may encroach on the intestinal lumen and cause obstruction to the passage of intestinal contents or they may lead to diverticulitis and perforation of the diverticulum. If the enterolith is expelled into the lumen, it may become impacted in a distal portion of the small intestine and cause obturation obstruction leading to local ischaemia and perforation. ${ }^{10}$

The case described in this communication is the first report of small bowel obstruction with multiple perforations caused by an enterolith not

\section{References}

1. Slater, S.N. Perforation and obstruction by enterolith complicating jejunal diverticulosis. Br J Surg 1953, 41: 60-62.

2. Bewes, P.C., Haslewood, G.A.D. \& Roxburgh, R.A. Bile acid enterolith and jejunal diverticulosis. $B r J$ Surg 1966, 53: $709-711$.

3. Walker, W.F., Kerr, G. \& MacDonald, J.S. Enterolith ileus. Br J Surg 1960, 48: 132-145.

4. Goldstein, S.S., Lewis, J.H. \& Rothstein, R. Intestinal obstruction due to bezoars. Am J Gastroenterol 1984 79: 313-318.

5. DeBakey, M. \& Ochsner, A. Bezoars and concretions - a comprehensive review of the literature with analysis of 303 collected cases and a presentation of 8 additional cases. Surgery 1939, 4: 934-963.

6. Liu, P.H.W. \& Ho, H.L. Ginger and drug bezoars induced small bowel obstruction. $J R$ Coll Surg Edinb 1983, 28: 397-398. associated with any gastrointestinal pathology that could explain its formation. It was probably due to a disordered intestinal motility. Stasis, caused partly by the presence of undigested vegetable material and partly by disordered jejunal peristal$\mathrm{sis}^{2,3}$ promotes the accumulation of bile and the multiplication of bacteria. The bacteria convert cholic acid to insoluble deoxycholic acid and also split glycine and taurine from the bile salts resulting in the precipitation of these materials around some nucleus of undigested vegetable material.

In this case presented, the ischaemic perforations of the small bowel appear to have developed at decubital ulcers produced at sites of earlier impactions proximal to the final one. The enterolith was disimpacted proximally by milking towards the lumen of the perforated segment of small bowel for its removal with the specimen.

If at laparotomy the small bowel obstruction is uncomplicated by necrosis and perforation, the enterolith should be broken up manually and the fragments milked distally into the colon. ${ }^{7}$ When this is unsuccessful, the enterolith should be milked proximally and removed through an enterotomy, performed in a segment of normal bowel where a satisfactory closure can be obtained. In cases where a thin-walled diverticulum gave origin to the enterolith and bears external evidence of delivery or doubtful viability, it should either be invaginated into the lumen of the gut or imbricated with seromuscular sutures. ${ }^{12}$ The presence of a second enterolith, though exceedingly rare, ${ }^{7}$ and 'gallstone ileus' should also be excluded. When bulky gastric tumours are resected, the small bowel must be examined carefully for intraluminal obstructing enteroliths (gastric cancer bezoars), which might, if left, give rise to obstruction. ${ }^{11}$

\section{Acknowledgement}

The secretarial work of Miss Lesley Cook is gratefully acknowledged.

7. Shocket, E. \& Simon, S.A. Small bowel obstruction due to enterolith (bezoar) formed in a duodenal diverticulum: a case report and review of the literature. Am J Gastroenterol 1982 , 77: $621-624$

8. Rumley, T.O., Hocking, M.P. \& King, C.E. Small bowel obstruction secondary to enzymic digestion of a gastric bezoar. Gastroenterology 1983, 84: 627-629.

9. Maull, K.I., Nicholson, B.W. \& Mendez-Picon, G. Jejunoileal diverticulosis. South Med J 1981, 74: 792-795.

10. King, P.M., Bird, D.R. \& Eremin, O. Enterolith obstruction of the small bowel. $J$ R Coll Surg Edinb 1985, 4: 269-270.

11. Deutsch, A.A., Gutman, H., Mor, C. \& Reiss, R. Gastric cancer bezoar. J R Coll Surg Edinb 1987, 32: 165-166.

12. Ottinger, L.W. \& Carter, E.L. Obturation of the ileum by a jejunal diverticular enterolith. Gastroenterology 1975, 68: 1596-1597. 